\section{Ingeniería}

\section{CONTENIDO}

\section{Artículos}

1. Sobre la redefinición del kilogramo Becerra, Luis; Hernández, Ignacio

2. Desarrollo y validación de un método de calibración de analizadore

de gases para vehículos con motores de encendido por chispa De Lemos, Leonora.

3. Validación de un método matemático para la calibración de frenómetros para motocicletas y para vehículos livianos Solano, Paula.

4. Propuesta para establecer las competencias técnicas para el proceso Metrología de México.. Herrera, Raúl; Mercader, Flora.

5. Aproximaciones y resultados para la medición práctica de iluminancia Marín, Luis Diego.

\section{Notas técnicas}

1. Resultados de la intercomparación costarricense de

calibración de cronómetro

Castro, Isabel; Sánchez, Harold.

2. Metodología para evaluar la competencia técnica utilizando rondas

de comparación internas, un ejemplo para termometría

Paniagua, Carlost.

Análisis de la ley de cudrado inverso para medición

práctica de iluminancia.

Marín, Luis Diego.

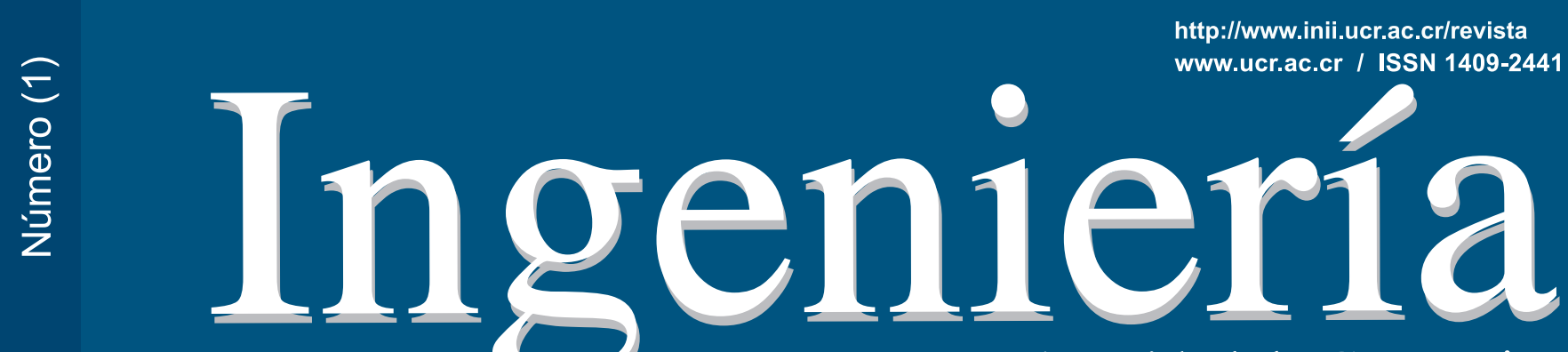

Revista de la Universidad de Costa Rica ENERO/ JULIO 2009 - VOLUMEN 19 - Número (1)

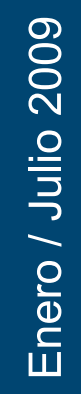

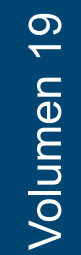

Normas

Normas para la presentación de artículos a la Revista Ingeniería.
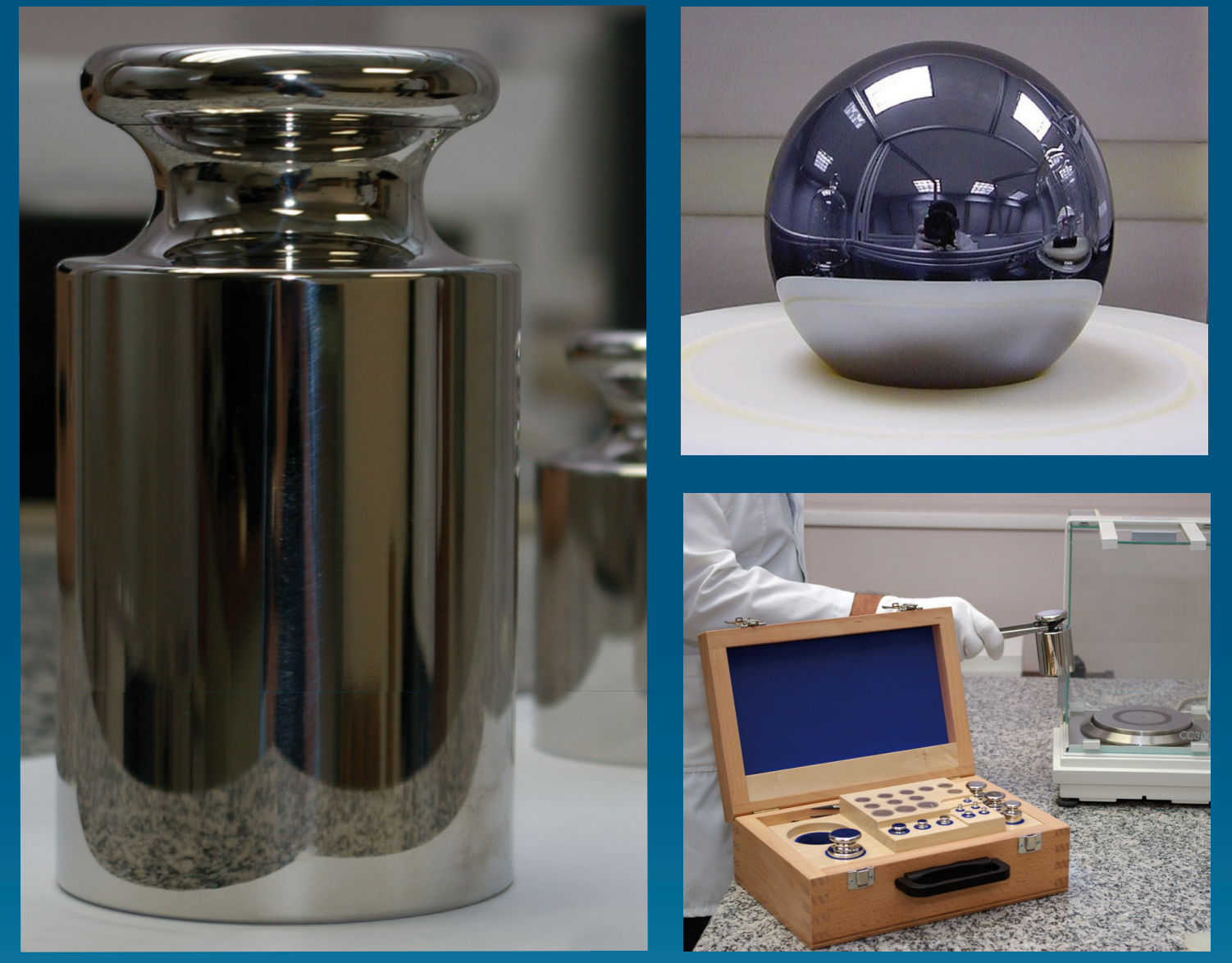

Metrología I 


\title{
SOBRE LA REDEFINICIÓN DEL KILOGRAMO
}

\author{
Luis Omar Becerra Santiago
} Ignacio Hernández Gutiérrez.

\begin{abstract}
Resumen
En la víspera de la posible redefinición de cuatro de las siete unidades de base del Sistema Internacional de Unidades (SI): kilogramo, ampere, kelvin, $\mathrm{y} \mathrm{mol}^{1}$ correspondientes a las siguientes magnitudes de base: masa, corriente eléctrica, temperatura termodinámica, y cantidad de sustancia, se hace necesario mencionar, objeto de este trabajo, los avances en la posible nueva definición de la unidad de masa, el kilogramo.
\end{abstract}

Palabras clave: kilogramo, Sistema Internacional de Unidades, redefinición del kilogramo.

\begin{abstract}
On the eve of a possible redefinition of four of the seven base units of the International System of Units (SI): kilogram, ampere, Kelvin, and mole ${ }^{1}$ corresponding to the following base quantities: mass, electric current, thermodynamic temperature, amount of substance; it is necessary to mention, subject of this paper, the progress on the new definition of the unit of mass, the kilogram.
\end{abstract}

Key words: kilogram, International System of Units, redefinition of the kilogram.

Recibido: 07 de noviembre del 2008 • Aprobado: 03 de diciembre del 2008

\section{INTRODUCCIÓN}

El Sistema Internacional de Unidades (SI) está formado por siete unidades de base: el metro, el kilogramo, el segundo, el ampere, el kelvin, la mol y la candela, correspondientes a las siete magnitudes de base: longitud, masa, tiempo, corriente eléctrica, temperatura termodinámica, cantidad de sustancia e intensidad luminosa.

En la 94 ${ }^{\mathrm{a}}$ Reunión del CIPM (Comité Internacional de Pesas y Medidas) realizada en octubre del 2005 se adoptó la recomendación de preparar los pasos para la redefinición del kilogramo, el ampere, el kelvin y la mol, de tal manera que estas unidades estén ligadas a valores conocidos de constantes fundamentales. En el caso particular de la unidad de masa, el kilogramo, podría ser redefinido en función de la constante de Planck $h$, o bien, del número de Avogadro $N_{A}$.

\section{DEFINICIÓN ACTUAL DEL KILOGRAMO}

La definición actual del kilogramo proviene de la propuesta de la Academia de Ciencias de Francia aprobada por la Asamblea Nacional y a encargo del rey Luis XVI a un grupo de miembros de la misma Academia de Ciencias (entre otros Legendré, Lavoisier, Coulomb, Borda, Berthollet, Lagrange, Delambre, Lefëvre-Gineau, Mechain), la formación de un "sistema métrico decimal" que posteriormente se convertiría en el Sistema Internacional de Unidades SI. La idea original de los comisionados del rey fue crear una unidad de 
masa que pudiera ser conocida como la "grave". Por definición ésta sería la masa de un litro de agua en su punto de congelación (esencialmente lo que ahora es un kilogramo). La definición fue materializada en un artefacto como patrón de masa (BIPM, The name "kilogram") ${ }^{1}$.

Después de la Revolución Francesa, el nuevo gobierno Republicano retomó la idea del sistema métrico pero realizó cambios significativos, por ejemplo, como muchas mediciones de aquella época involucraban mediciones de masa mucho más pequeñas que el actual kilogramo, ellos decidieron que la unidad de masa debía ser la "gramme" (gramo) una milésima parte de la "grave".

De cualquier manera, como el patrón de un gramo fue difícil de utilizar así como de establecer, decidieron que la nueva definición debía materializarse como un artefacto de un kilogramo que fue depositado en los archivos de Francia.

Este artefacto se convirtió en el "kilogramo de los archivos". En 1875 la unidad de masa fue redefinida como el "kilogramo", materializado como un nuevo artefacto cuya masa fuese esencialmente igual a la masa del "kilogramo de los archivos" (Davis, 2003).

La definición actual de la unidad de masa fue establecida en la $3^{\text {a }}$ Conferencia General de Pesas y Medidas (CGPM) en 1901 (BIPM, 1998):

"El kilogramo es la unidad de masa; es igual a la masa del prototipo internacional del kilogramo".

La unidad de masa, el kilogramo prototipo internacional $\mathcal{K}$ está conservado en la Oficina Internacional de Pesas y Medidas (BIPM), es un cilindro de aproximadamente $39 \mathrm{~mm}$ de diámetro por aproximadamente $39 \mathrm{~mm}$ de altura fabricado de una aleación para la cual la fracción de masa de platino es del $90 \%$ y la fracción de masa de iridio es del $10 \%$. Las masas de los patrones secundarios de $1 \mathrm{~kg}$ de la misma aleación o de acero inoxidable son comparadas contra la masa del prototipo mediante el uso de una balanza con una incertidumbre relativa del orden de 1 x 10-9.

Debidoaquelamasadelprototipointernacional $\mathcal{K}$ se incrementa por aproximadamente 1 parte en $10^{9}$ por año debido a la inevitable acumulación de contaminantes en su superficie, el CIPM declara que para la realización práctica del kilogramo la masa de referencia del prototipo internacional $\mathcal{K}$ es aquella después de haber sido aplicado un procedimiento específico de limpieza y de lavado (Mills, Mohr, Quinn, Taylor \& Williams, 2006).

La masa de referencia definida es utilizada para calibrar los patrones nacionales fabricados en la aleación de platino iridio. Para el caso de la calibración de patrones de masa fabricados en acero inoxidable, la incertidumbre es del orden de $1 \times 10^{-8}$ debido a la contribución de incertidumbre del empuje del aire.

\section{ESCALA NACIONAL DE MASA}

La escala nacional de masa de México, esta ligada al prototipo internacional del kilogramo $\mathcal{K}$ a través del prototipo de platino iridio identificado con el No.21,k21 (Figura 2), en donde su valor ha sido determinado en las verificaciones periódicas organizadas por el BIPM y establecidas por la CGPM (Girad, 1994).

El Centro Nacional de Metrología de México (CENAM) es el responsable de diseminar la exactitud de la unidad de masa hacia los usuarios en general, y esto se realiza principalmente con la generación de la escala nacional de masa, que consiste en patrones de masa de valor nominal de $1 \mathrm{mg}$ a $1000 \mathrm{~kg}$ cuyo valor de masa es referido al k21 y que mediante dichos patrones se calibran pesas e instrumentos para pesar que son los que se utilizan para las mediciones de masa en las actividades económicas, productivas, educativas, sociales, de investigación y de salud del país (metrología industrial, legal y científica).

La confiabilidad de las mediciones de masa actualmente es validada mediante la participación del CENAM en las comparaciones internacionales, varias de ellas son comparaciones clave organizadas por el Comité Consultivo para la Masa (CCM) del Comité Internacional de Pesas y Medidas (CIPM) y cuyos resultados pueden ser consultados en el apéndice B del Arreglo de Reconocimiento Mutuo (MRA, por sus siglas en inglés) en: http://kcdb.bipm.org/appendixB/. 


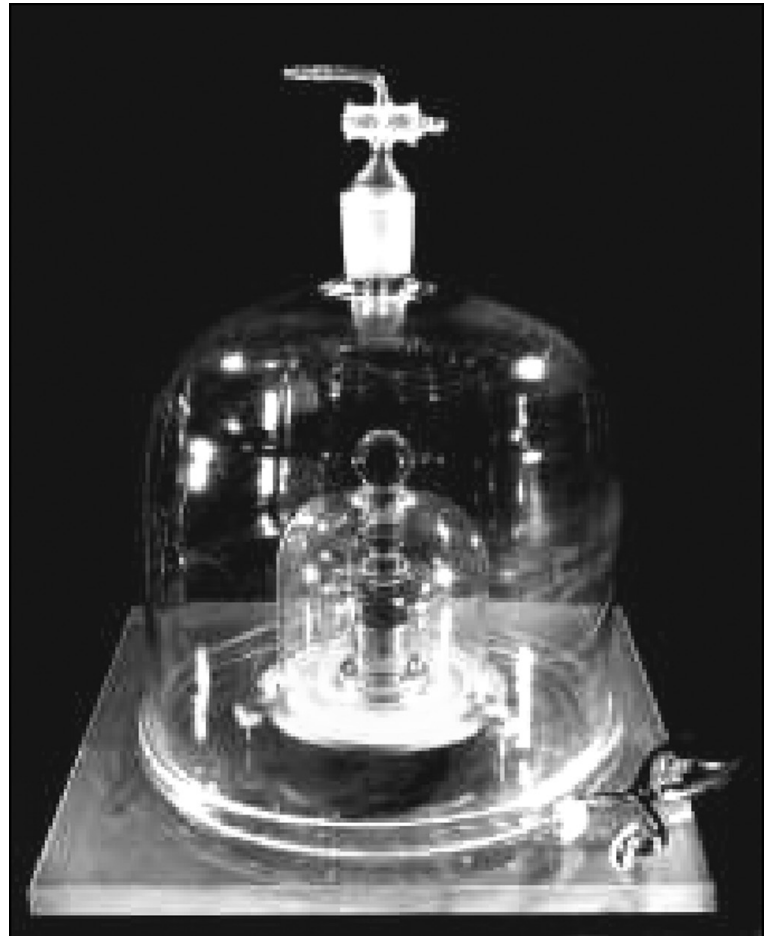

Figura 1. Prototipo Internacional del kilogramo $\mathcal{K}$, fabricado en la década de 1880 y autorizado como el prototipo internacional en 1889. Fue depositado en el Pavillon de Breteuil el 28 de septiembre de 1889. Fuente: (Esta fotografía fue reproducida con el permiso del BIPM, el cual retiene internacionalmente protegidos todos los derechos de autor).

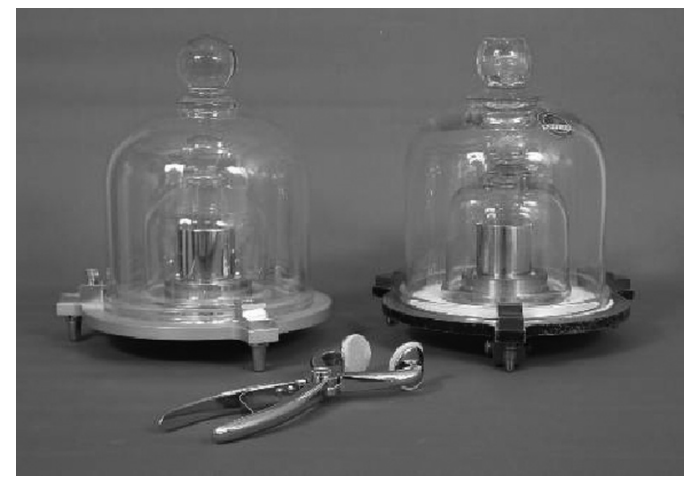

Figura 2. Patrón Nacional de Masa de México, prototipo del kilogramo identificado como No. 21 (derecha, en México desde enero de 1892), y prototipo del kilogramo identificado como No. 90 (izquierda, en México desde diciembre del 2004).

Fuente: (CENAM). 


\section{DERIVA DEL PROTOTIPO INTERNACIONAL DE KILOGRAMO K}

La deriva del prototipo internacional del kilogramo $\mathcal{K}$ únicamente puede ser estimada en función de la comparación en masa de este contra las copias fabricadas también en platino iridio, copias oficiales pertenecientes al BIPM o los patrones nacionales de los diferentes países que cuentan con este tipo de patrones.

En la tercera verificación periódica de los prototipos nacionales del kilogramo (Girard, 1994) se aprecia que la deriva anual de los patrones de platino iridio es en promedio de entre $0,25 \mu \mathrm{g} / \mathrm{año}$ a $0,9 \mu \mathrm{g} / \mathrm{año}$ en función del uso y los cuidados de los que fue objeto (Figura 3 ).

La primera verificación de los prototipos de platino iridio fue realizada de 1899 a 1911; la segunda verificación inició en 1939 pero fue interrumpida por la Segunda Guerra Mundial y fue reanudada en 1946 para concluir en 1953. La tercera y última verificación de los prototipos de platino iridio inició en 1988 y finalizó en 1992.

La incertidumbre resultante de la tercera verificación de los prototipos de platino iridio fue de $2,3 \mu \mathrm{g}$, sin embargo este valor de incertidumbre fue estimado únicamente mediante inferencia estadística (contribución tipo A) (BIPM et al, 1995).

Esta situación es muy importante para el futuro de la definición de la unidad de masa, debido a que si se considera la deriva anual promedio estimada para los patrones de platino iridio y el periodo promedio en el que se han realizado las verificaciones de este tipo de patrones, aunado a que la referencia (la definición de la unidad de masa) el prototipo internacional del kilogramo $\mathcal{K}$ es un artefacto que al igual que el resto de las copias (prototipos de platino iridio) está expuesto tanto a contaminación como a desgaste, la referencia para la unidad de la masa en el Sistema Internacional de Unidades no es fija como actualmente es el caso para otras unidades del SI, (puede tener una variación en masa de hasta $50 \mu \mathrm{g}$ en un siglo), por ejemplo la unidad del tiempo, el segundo, está definido en función del periodo de la transición hiperfina del átomo de Cesio 133, para lo cual se fijó el valor de la frecuencia de transición del átomo de Cesio 133 en la $2^{\text {a }}$ Reunión de la CGPM (CGPM, 1960), o la definición de la unidad de longitud, el metro el cual está definido en función de la velocidad de la luz $\mathrm{c}_{o}$, para lo cual se fijó el valor de $\mathrm{c}_{o}$ en la $15^{\mathrm{a}}$ Reunión de la CGPM (CGPM, 1975), por mencionar sólo dos ejemplos.

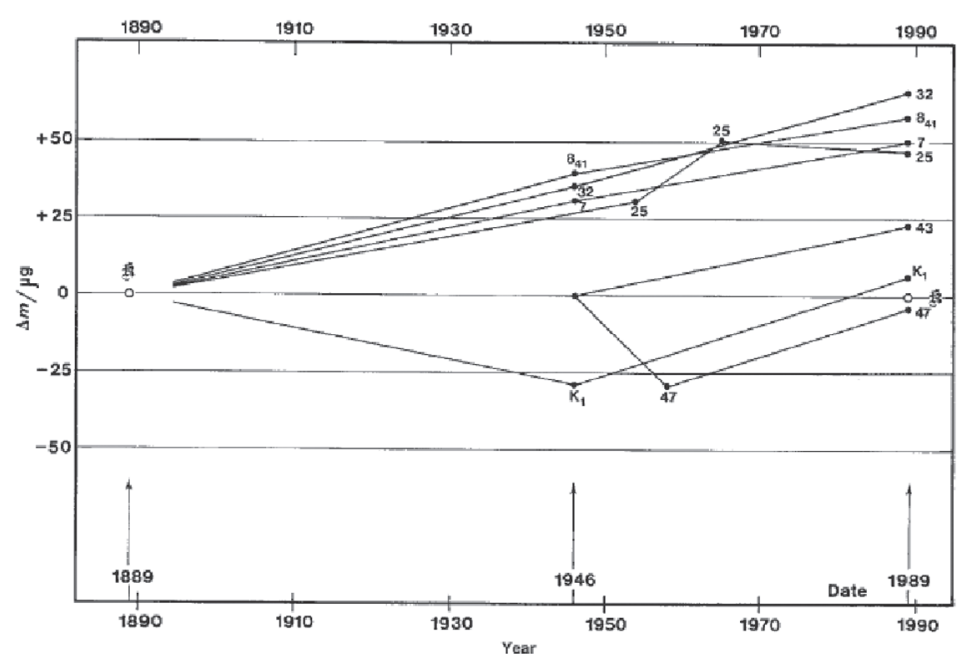

Figura 3. Cambios relativos en masa de seis copias oficiales (k1, y las copias No. 7, 8(41), 32, 43 y 47) con respecto al prototipo internacional del kilogramo K. Fuente: (Girard, 1994). 
El hecho de definir las unidades del SI en función de constantes físicas o de constantes de la naturaleza disminuye la deriva del valor de referencia y la incertidumbre asociada a esta deriva, que por otro lado, para el caso específico de la magnitud masa, actualmente no es considerada en la estimación de la incertidumbre de los prototipos de platino iridio (en las verificaciones internacionales periódicas).

\section{PROYECTOS PARA LA REDEFINICIÓN DEL KILOGRAMO}

Con la intención de que la unidad de la magnitud de masa esté relacionada a alguna constante física o constante de la naturaleza se han desarrollado algunos proyectos primero con la intención de monitorear la deriva del prototipo internacional del kilogramo $\mathcal{K}$ y posteriormente se ha pensado en cambiar la actual definición de tal manera que el kilogramo se defina en función de dichas constantes.

Con esta intención se han iniciado algunos proyectos como Levitación magnética de un superconductor cuya idea original corresponde a Sullivan y Frederich como una posibilidad de realizar el ampere (Sullivan \& Frederich, 1977), La balanza de tensión eléctrica desarrollado por la Universidad de Zagreb, Croacia (Bego, Butorac \& Poljancic, 1995) y el PTB de Alemania (Funk \& Sienknecht, 1991), el Conteo de iones de oro desarrollado por el PTB de Alemania (Gläser, 2003); sin embargo los dos proyectos que van a la vanguardia sobre la redefinición del kilogramo son La balanza de Watt y del Número de Avogadro.

\subsection{La balanza de Watt}

El experimento que se realiza en la balanza de Watt consiste en comparar la potencia eléctrica con la potencia mecánica (Eichenberger, Jeckelmann \& Richard, 2003).

El experimento está compuesto de dos partes, la primera parte consiste en aplicar una intensidad de corriente $I$ a parte de una bobina suspendida del brazo de una balanza (de brazos iguales), que al ser introducida en un campo magnético horizontal $B$ (y perpendicular a la bobina) genera una fuerza $F$. Esta fuerza $F$ es equilibrada al colocar en el otro brazo de la balanza una masa $\mathrm{m}_{s}$ sometida a aceleración de la gravedad $g$, ver Figura 4.

En la segunda parte del experimento, la bobina es movida a una velocidad constante $\mathrm{v}$ en la dirección perpendicular al flujo $B$, induciéndose en la bobina una tensión eléctrica $U$, la cual es medida. La expresión para la determinación de la masa de un objeto mediante la balanza de Watt, derivada a partir de las dos partes del experimento, queda en la ecuación (1).

Donde,

$m_{s}=h \cdot \frac{K_{J-90}^{2} \cdot R_{K-90} \cdot U \cdot I}{4 \cdot g \cdot v}$

$h \quad$ constante de Planck ${ }^{2}$

$K_{J-90} \quad$ constante de Josephson

$R_{K-90} \quad$ constante de von Klitzing

$U$ tensión eléctrica inducida en una bobina móvil medida en términos del Efecto Josephson y la constante de Josephson, $K_{J-90}$

I intensidad de corriente eléctrica inducida en la bobina móvil y medida en términos del Efecto Josephson, el efecto cuántico Hall, la constante de Josephson $K_{J-90}$ y la constante de von Klitzing $R_{K-90}$

$g \quad$ valor de la aceleración de la gravedad local

$v \quad$ velocidad de la bobina móvil

Este proyecto se encuentra activo actualmente en el NPL-Reino Unido3, en el NIST-Estados Unidos, en METAS-Suiza, LNE-Francia y el BIPM-Internacional.

\subsection{El proyecto del Número de Avogadro}

La idea principal de este proyecto consiste en definir el kilogramo como la masa de un número definido de átomos de algún material, por ejemplo, Carbono 12. 


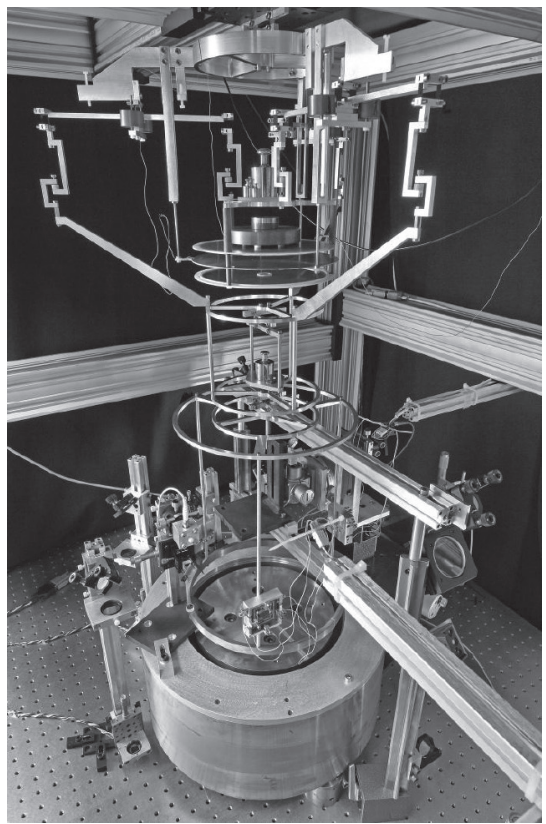

Figura 4. Balanza de Watt del BIPM, ubicada en el Pavillon de Breteuil, Sevrés Francia.

Fuente: (Esta fotografía fue reproducida con el permiso del BIPM, el cual retiene internacionalmente protegidos todos los derechos de autor).

La siguiente expresión define el kilogramo,

$$
1 \mathrm{~kg}=10^{3} N_{A} u
$$

Donde,

$N_{A}$, constante de Avogadro o número de Avogadro

$u \quad$ unidad atómica de masa definida como,

$u=\frac{1}{12} m\left({ }^{12} C\right)$

$m\left({ }^{12} C\right)$ masa de un átomo de carbono 12.

De la ecuación (2) se puede ver que para obtener una masa de un kilogramo de Carbono 12 es necesario reunir un número definido de átomos de dicho elemento,

$$
\left(\frac{N_{A}}{0,012}=5,0184512725 \times 10^{25}\right)
$$

El número de Avogadro es una constante fundamental que relaciona la escala atómica de cualquier magnitud con la escala macroscópica (Becker, 2003; Peuto, 2002),

$$
N_{A}=\frac{M}{A}=\frac{V_{0}}{V_{a}}
$$

Donde:

$M \quad$ masa molar

A masa atómica

$V_{0} \quad$ volumen molar

$V_{a} \quad$ volumen atómico 
La determinación experimental del número de Avogadro vía el silicio, está basada en mediciones a esferas de monocristal de silicio de aproximadamente $1 \mathrm{~kg}$ (Figura 5).

El número de Avogadro puede ser determinado mediante la medición de la masa $m$ y el volumen $V$ de estas esferas de monocristal de silicio. Como la masa molar $M(S i)$ y volumen molar $V_{0}$ del silicio son conocidos para la celda unitaria (con $\mathrm{n}=8$ átomos) el Número de Avogadro se calcula mediante la siguiente expresión,

$$
N_{A}=n \frac{M(S i)}{m} \frac{V}{V_{0}}
$$

que se deriva de la ecuación (4) con las ecuaciones (6) y (7).

$$
V_{0}=\frac{M(S i)}{m} V
$$

$V_{a}=\frac{V_{0}}{n}$

El proyecto de Avogadro es realizado bajo la supervisión del Grupo de Trabajo de la

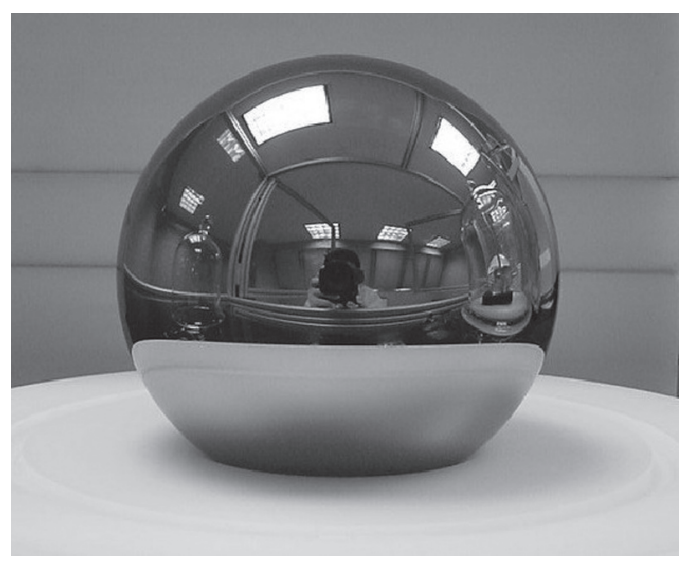

Figura 5. Esfera de monocristal de silicio. Fuente: (CENAM).
Constante de Avogadro (WGAC) del CCM. Este proyecto es un esfuerzo multinacional que esta estructurado de la manera que se muestra en el Cuadro 1.

Una vez conocido $N_{A}$ y los parámetros del material $(\mathrm{Si})$, la evaluación de la masa de una esfera de silicio se puede obtener mediante mediciones dimensionales de la esfera, ver la ecuación (8).

$$
m_{s}(S i)=\frac{\pi(1+k) A_{r}(S i) M_{0}}{12 \sqrt{2} N_{A}}\left(\frac{d_{s}(S i)}{d_{220}(S i)}\right)^{3}
$$

Donde:

$m_{s}(\mathrm{Si})$ masa de la esfera de monocristal de silicio

$(1+k)$ factor de masa molar

$A_{r}(\mathrm{Si})$ masa atómica relativa del silicio

$M_{0} \quad$ constante de masa molar

$d_{s}(S i)$ diámetro medio de la esfera

$d_{220}(S i)$ distancia de la estructura $\{220\}$ del monocristal de silicio

\subsection{Discrepancia entre los resultados obtenidos mediante el proyecto de la balanza de Watt y mediante el proyecto de Avogadro}

Actualmente la incertidumbre declarada para la determinación de $h$ en el experimento de la Balanza de Watt es de 8,7 x $10^{-8}$, y la incertidumbre reportada para la determinación de $h$ para el Proyecto de Avogadro Internacional es de 3,2 x $10^{-7}$, sin embargo existe una inconsistencia entre ellos al relacionar la constante de Planck $h$ con el número de Avogadro $N_{A}$ mediante la ecuación (9) del orden de $1 \times 10^{-6}$, lo cual significa que existe un error sistemático en al menos uno de los dos experimentos que se debe corregir para disminuir esta discrepancia (Taylor \& Mohr, 1999).

$h=\frac{c_{0} A_{r}(e) M_{0} \alpha^{2}}{2 R_{\infty} N_{A}}$ 
Donde,

$\begin{array}{ll}c_{0} & \text { velocidad de la luz en el vacío } \\ A_{r}(e) & \text { masa atómica relativa del electrón } \\ M_{0} & \text { constante de masa molar } \\ \alpha & \text { constante de la estructura fina } \\ R_{\infty} & \text { Constante de Ridberg }\end{array}$

\section{RECOMENDACIÓN 1 (CI-2005) DEL CIPM SOBRE LA REDEFINICIÓN DEL KILOGRAMO, EL AMPERE, EL KELVIN Y LA MOL EN TÉRMINOS DE CONSTANTES FUNDAMENTALES}

Con relación al posible cambio de algunas de las unidades de base del SI, el CIPM recibió las recomendaciones de los Comités Consultivos involucrados en dichos cambios. Estas recomendaciones, fueron consideradas en la recomendación emitida por el CIPM.

En lo que se refiere al posible cambio de la definición del kilogramo, el CCM emitió la recomendación G1 (2005) al CIPM en la cual se menciona que la discrepancia existente entre los resultados de las mediciones experimentales de $h$ sea resuelta (sección 5.3) y que la incertidumbre para la realización del kilogramo no exceda en $2 \times 10^{-8}$ al nivel de $1 \mathrm{~kg}$, lo cual implica que la incertidumbre relativa para la definición de $h$ sea de $2 \times 10^{-8}$ (CCM, 2005; CCU, 2005).
De la misma manera el Comité Consultivo para las Unidades (CCU) en la Recomendación U1 (2005), solicita al CIPM que apruebe en principio la preparación de las nuevas definiciones y realizaciones prácticas del kilogramo, el ampère y el kelvin, de modo que si los resultados de las mediciones experimentales son aceptables y acordadas entre los diferentes Comités Consultivos y otros organismos relevantes, el CIPM pueda preparar propuestas a los Estados Miembros del Tratado del Metro a tiempo para su posible adopción en la $24^{a}$ reunión de la CGPM en 2011 (Mills, Mohr, Quinn, Taylor, \& Williams, 2006; CCM, 2005; CCU, 2005; CCEM, 2005).

En la misma recomendación el CCU recomienda al CIPM preparar una Resolución a ser puesta en la agenda de la $23^{a}$ Reunión de la CGPM a realizarse en el 2007 que alerte a los Estados Miembros de estas actividades.

De la recomendación 1 del CIPM (CI-2005), a continuación se escribe un extracto,

Pasos preparatorios hacia nuevas definiciones del kilogramo, el ampere, el kelvin y la mol en función de constantes fundamentales

- aprueba en principio la preparación de las nuevas definiciones y realizaciones prácticas del kilogramo, el ampere y el kelvin de manera que si los resultados de las mediciones experimentales de los

Cuadro 1. Estructura del proyecto de Avogadro.

Subproyecto

Densidad absoluta

Densidad relativa

Parámetro absoluto de la estructura

Esferas, base de datos

Masa molar

Parámetro relativo de la estructura

Material

Masa

Superficie

* Laboratorios piloto del proyecto.

\section{Institutos Participantes}

NMIJ-Japón*, PTB-Alemania, CSIRO-Australia

PTB-Alemania*, NMIJ-Japón, IMGC-Italia

IMGC-Italia*, PTB-Alemania

CSIRO-Australia*, IRMM-Europa, BIPM-Internacional

IRMM-Europa*

NIST-E.U.*, PTB-Alemania

PTB-Alemania*, VITCON/IKZ-Alemania, NIST-E.U., NMIJ-Japón, IRMM-Europa

PTB-Alemania*, BIPM-Internacional, NPL-Reino Unido

NPL-Reino Unido*, PTB-Alemania, NMIJ-Japón, CSIRO-Australia

Fuente: (Peuto, 2002). 
próximos años son de hecho aceptables y existiendo el acuerdo en los diferentes Comités Consultivos y otras organizaciones relevantes, el CIPM puede preparar propuestas para ser expuestas a los Estados Miembros de la Convención del Metro a tiempo para su posible adopción en la $24^{\mathrm{a}}$ CGPM a realizarse en 2011.

invita a todos los Comités Consultivos

- en particular al CCM, CCEM, CCQM y CCT a considerar las implicaciones de cambiar las definiciones de las unidades de base mencionadas anteriormente del SI, y a presentar un informe al CIPM antes del junio del 2007.

- supervisar estrechamente los resultados de nuevos experimentos relevantes de las nuevas posibles definiciones, identificar las posibles nuevas condiciones a ser cumplidas antes de proceder al cambio de las definiciones, y a considerar en particular la alternativa de redefinición de las unidades mencionadas previamente.

- solicitar información a nivel mundial de la comunidad científica y técnica sobre la materia.

De esta recomendación se puede apreciar que muy posiblemente en el 2011 pueda ser redefinido el kilogramo al igual que el ampere, el kelvin y la mol.

\section{ALTERNATIVAS PARA LA NUEVA DEFINICIÓN DE LA UNIDAD DE MASA, EL KILOGRAMO}

La tendencia del CCU (Comité Consultivo para las Unidades) es que la nueva definición del kilogramo este referida a la constante de Planck. Algunas de las posibles definiciones para el kilogramo en función de la constante de Planck son las siguientes (Mills, Mohr, Quinn, Taylor \& Williamns, 2005),

i. El kilogramo es la masa de un cuerpo en reposo tal que el valor de la constante de Planck $h$ es exactamente igual a 6,626069
$311 \times 10^{-34}$ joule segundo.

ii. El kilogramo es la masa de un cuerpo en reposo cuya energía equivalente corresponde exactamente a la frecuencia de [(299792 $458)^{2}$ / 6626069311$] \times 10^{43}$ hertz.

iii. El kilogramo es la masa de un cuerpo cuya longitud de onda Broglie es exactamente 6,626069 $311 \times 10^{-34} \mathrm{~m}$ cuando este se mueve a una velocidad de exactamente un metro por segundo.

La propuesta ii fija el valor de $h$ mediante la combinación de la relación de Einstein $\mathrm{E}=\mathrm{mc}^{2}$ y la relación $E=h v$ aplicada por primera vez por Planck para la emisión y absorción de la radiación y posteriormente por Einstein para la energía de los fotones, mientras la propuesta iii fija el valor de $h$ mediante de la relación de Broglie $\lambda=h / p=h / m v$.

Otra posibilidad, es que la definición de la unidad de masa este ligada a la constante de Avogadro $N_{A}$.

La constante de Avogadro (en $\mathrm{mol}^{-1}$ ) esta definida como $N_{A}=M(X) / m(X)$, donde $M(X)$ es la masa molar de la entidad $X$ (e. g. la masa por cantidad de sustancia de $X$, en $\mathrm{kg} \mathrm{mol}^{-1}$ ) y $m(X)$ es la masa de $X$ (en $\mathrm{kg}$ ). Por lo tanto,

a. el número de entidades de un mole de $X$ es $\left(N_{A} \mathrm{~mol}\right)$,

b. $\left(N_{A} \mathrm{~mol}\right) m\left({ }^{12} C\right)=0,012 \mathrm{~kg}$ exactamente, $\mathrm{y}$

c. La masa molar del carbono $12 M\left({ }^{12} \mathrm{C}\right)$, es exactamente igual a $0,012 \mathrm{~kg} \mathrm{~mol}^{-1}$

De lo anterior se derivan las siguientes posibilidades que podrían ser la nueva definición de la unidad de masa el kilogramo,

iv. El kilogramo es la masa de un cuerpo en reposo tal que el valor de la constante de Avogadro $N_{A}$ es exactamente igual a 6,022 $141527 \times 10^{23} \mathrm{~mol}^{-1}$

v. El kilogramo es la masa de exactamente 5,018451 $2725 \times 10^{25}$ de átomos libres de carbono 12 en reposo y en su estado base.

vi. El kilogramo es la masa de exactamente $\left(6,022141527 \times 10^{23} / 0,012\right)$ átomos libres de carbono 12 en reposo y en su estado base. 


\section{IMPACTO DEL CAMBIO DE LA DEFINICIÓN DE LA UNIDAD DE MASA A LOS USUARIOS EN GENERAL}

Una vez que se realice el cambio en la definición del kilogramo, los patrones de Pt-Ir pertenecientes a los diferentes países (Patrones Nacionales), podrán ser enviados a alguno de los Institutos en donde se encuentre activo el proyecto seleccionado para reproducir al kilogramo (por ejemplo la Balanza de Watt), a ser recalibrados periódicamente y de esta manera obtener trazabilidad en el valor de masa de dichos patrones nacionales y generar la escala de masa en los diferentes países.

El CENAM al igual que el resto de los Institutos Nacionales de Metrología, en lo que se refiere a diseminar la unidad de masa, continuará con la utilización de dichos prototipos de platino iridio (k21 y k90 para el caso de México), que se ha demostrado que son los patrones que menor deriva presentan en relación a otros materiales, por ejemplo acero inoxidable, como primer eslabón en la cadena de trazabilidad de las mediciones de masa del país.

A partir de dichas referencia (Patrón Nacional de Masa) se realizará la transferencia de exactitud hacia los patrones de acero inoxidable y posteriormente la generación de la escala nacional de masa y el resto de actividades que actualmente se realizan de la misma manera que al momento, por lo que el efecto del cambio de la definición del kilogramo no será percibido significativamente en los usuarios finales de la metrología de masa.

No obstante el hecho de que la unidad de masa ya no este referida a un artefacto expuesto a contaminación y/o a daño físico y que el kilogramo pueda ser reproducido libremente eliminando la incertidumbre de la deriva de la definición actual, es una de las ventajas que ofrece este cambio.

La definición de la unidad de masa del SI, el kilogramo como la masa igual a la del prototipo internacional es la que mas dificultades ha presentado para que la ciencia pueda definirla como una función de constantes fundamentales, y estamos muy probablemente en la víspera del cambio de la misma.

\section{CONCLUSIONES}

1. Con el cambio de la definición de la unidad de masa, el kilogramo ligada a la constante de Planck o al Número de Avogadro, se reduciría la incertidumbre de la deriva asociada al valor de la definición del kilogramo, que actualmente no es considerada. Con la nueva definición aun cuando aparentemente se incrementará la incertidumbre de calibración de los prototipos nacionales, en realidad la incertidumbre sería menor que la que se obtendría actualmente si se considerara la incertidumbre debido a la posible deriva del Prototipo Internacional del kilogramo $\mathcal{K}$.

2. Los usuarios de los valores de masa no se verán afectados por la nueva definición, porque la nueva definición del kilogramo será coherente con el valor actual del prototipo internacional del kilogramo $\mathcal{K}$ y de esta forma permanecerá la base para el sistema práctico mundial de las mediciones de masa.

\section{NOTAS}

1. En el Vocabulario Internacional de Metrología - Conceptos fundamentales y generales, y términos asociados (VIM), $1^{\text {a }}$ Edición en Español 2008, JCGM 200:2008, se define a la "cantidad de sustancia" como una de las magnitudes de base del SI y a la "mol" como su unidad. Sin embargo en Costa Rica mediante el decreto DE $\mathrm{N}^{\circ}$. 29660 MEIC, el nombre oficial para esta misma magnitud es oficialmente "cantidad de materia" y su unidad es la "mole".

2. Para consultar los valores recomendados para las diferentes constantes ver http://physics. nist.gov/cuu/Constants.

3. El experimento de la balanza de Watt del NPLReino Unido será transferido al NRC- Canadá en el transcurso del 2009. 


\section{REFERENCIAS BILBLIOGRÁFICAS}

Becker, P. (2003). Tracing the definition of the kilogram to the Avogadro constant using a silicon single crystal. Metrologia, 40(6), 366-375.

Bego, V., Butorac, J \& Poljancic, K. (1995 Apr). Voltage balance for replacing the kilogram. IEEE Trans. Instrum. Meas., 44(2), 579-82.

BIPM, IEC, IFCC, ISO, IUPAC, IUPAP \& OIML. (1995). Guide to the expression of uncertainty in measurement. Extraído de: http://www.bipm.org/en/publications/ guides/. Consultado en junio 2009.

BIPM.(1998).The International Systems of Units, $7^{a}$ edit. Organización Intergubernamental de la Convención del Metro. In: www.bipm.fr. Consultado en mayo 2008.

BIPM. The name "kilogram": a historical quirk. In: www.bipm.fr. Extraído en mayo 2008.

CCEM (Consultative Committee for Electricity and Magnetism). (2005 Mar). Report of the 24th meeting (17-18March 2005) to the International Committee for Weights and Measures.

CCM (Consultative Committee for Mass and Related Quantities). (2005 Apr). Report of the 9th meeting (28-29 April 2005) to the International Committee for Weights and Measures, www.bipm.fr/utils/common/pdf/ CCM9.pdf. Extraído en mayo 2008.

CCU (Consultative Committee for Units). (2005 Jul). Report of the 17th meeting (29 June 1 July 2005) to the International Committee for Weights and Measures, www.bipm.fr/ utils/common/pdf/CCU17.pdf. Consultado en mayo 2008.

CGPM (Conférence Génerale des Poids et Mesures). (1960). Resolución 6 de la $11^{a}$ Reunión de la CGPM. http://www.bipm.org/ en/CGPM/db/15/2/. Consultado el 8 de julio del 2009.
CGPM (Conférence Génerale des Poids et Mesures). (1975). Resolución 2 de la $15^{a}$ Reunión de la CGPM. http://www.bipm.org/en/CGPM/ db/15/2/. Consultado el 8 de julio del 2009.

CODATA (Comimmittee on Data for Science and Technology). CODATA Internationally recommended values of the fundamental physical constants. http://physics.nist/.gov/ cuu/Constants Consultado en mayo 2008.

Davis, R. (2003). The SI unit of mass. Metrologia, 40(6), 299-305.

Eichenberger, A., Jeckelmann, B. \& Richard, P. (2003). Tracing Planck's constant to the kilogram by electromechanical methods. Metrologia, 40(6), 356-365

Funk, T. \& Sienknecht, V. (1991 Apr). Determination of the volt with the improved PTB voltage balance. IEEE Trans. Instru. Meas., 40(2), 158-61.

Girard, G. (1994). International Report: The third periodic verification of the national prototypes of the kilogram (1988-1992). Metrologia, 31(4), 317-336.

Gläser, M. (2003). Tracing the atomic mass unit to the kilogram by ion accumulation. Metrologia, 40(6), 376-386.

Mills, I. M., Mohr, P. J., Quinn, T. J., Taylor, B. N. \& Williams, E. R. (2006). Redefinition of the kilogram, ampere, kelvin and mole: a proposed approach to implementing CIPM recommendation 1 (C1-2005). Metrologia, 43(3), 227-246.

Mills, I. M., Mohr, P. J., Quinn, T. J., Taylor, B. N. \& Williams, E. R. (2005). Redefinition of the kilo gram: a decision whose time has come. Metrologia, 42(2), 71-80.

Peuto, A. (2002 Oct). The Avogadro Constant: definition and determination. Conferencia de la Dra. Anna Peuto del IMGC-Italia en el CENAM Querétaro, México. 
Sullivan, D. B. \& Frederich, N. V. (1977 Jan). Can superconductivity contribute to the determination of the absolute ampere?. IEEE Trans. Magetics, 13(1), 396-9.

Taylor, B. N. \& Mohr, P. J. (1999). On the redefinition of the kilogram. Metrologia, 36(1), 63-64. www.bipm.fr/utils/common/ pdf/CCEM24.pdf. Consultado en mayo 2008.

\section{SOBRE LOS AUTORES}

\section{Luis Omar Becerra Santiago}

Maestro en Ciencias, Coordinador Científico de la División de Masa y Densidad
Centro Nacional de Metrología (CENAM). Carretera a los Cués en el kilómetro 4,5, Mpio. El Marqués, Querétaro, México

Teléfono: +52 4422110500 extensión 3602

Fax: +524422110568

Correo electrónico: lbecerra@ cenam.mx

\section{Ignacio Hernández Gutiérrez}

Ph. D.

Director del Área de Metrología Mecánica

Centro Nacional de Metrología (CENAM)

Carretera a los Cués en el kilómetro 4,5, Mpio. El

Marqués, Querétaro, México

Teléfono: +524422110570

Fax: +52 4422110568

Correo electrónico: ihernan@cenam.mx 